\title{
NOTE ON A SUBGROUP OF THE MODULAR GROUP'
}

\author{
M. NEWMAN AND J. R. SMART
}

Let $\Gamma$ denote the $2 \times 2$ modular group; that is, the multiplicative group of $2 \times 2$ rational integral matrices of determinant 1 in which a matrix is identified with its negative. Let $\Gamma(n)$ denote the principal congruence subgroup of $\Gamma$ of level $n$; that is, the totality of elements $a$ of $\Gamma$ such that

$$
a \equiv \pm\left(\begin{array}{ll}
1 & 0 \\
0 & 1
\end{array}\right) \quad(\bmod n)
$$

Let $G^{\prime}$ denote the commutator subgroup of $G, G$ any subgroup of $\Gamma$. Finally, let $\Gamma^{n}$ denote the fully invariant subgroup of $\Gamma$ generated by the $n$th powers of the elements of $\Gamma$. Then it is shown in [4] that

$$
\left(\Gamma^{2}\right)^{\prime} \supset \Gamma(6)
$$

and the same method can be used to show that

$$
\left(\Gamma^{3}\right)^{\prime} \supset \Gamma(6) \text {. }
$$

Hence

$$
\left(\Gamma^{2}\right)^{\prime} \cap\left(\Gamma^{3}\right)^{\prime} \supset \Gamma(6)
$$

and the question arises as to the precise relationship between $\left(\Gamma^{2}\right)^{\prime} \cap\left(\Gamma^{3}\right)^{\prime}$ and $\Gamma(6)$. The object of this note is to prove that these are in fact equal.

We set

$$
G=\left(\Gamma^{2}\right)^{\prime}, \quad H=\left(\Gamma^{3}\right)^{\prime}
$$

and break the proof up into several lemmas.

LEMMA 1. The group $G$ is a free group of rank 4 and of index 3 in $\Gamma^{\prime}$. The group $H$ is a free group of rank 5 and of index 4 in $\Gamma^{\prime}$.

Proof. It was shown in [1] that $\Gamma^{2}$ is the free product of two cyclic groups of order 3 , and $\Gamma^{3}$ the free product of three cyclic groups of order 2. Now J. Nielsen has shown [2] that the commutator subgroup of the free product of $k$ finite cyclic groups $G_{i}$ of order $m_{i}, 1 \leqq i \leqq k$ is a free group of rank

Received by the editors January 4, 1962.

1 The work of the first author was supported by the Office of Naval Research, and that of the second author by a Postdoctoral Fellowship from the National Science Foundation. 


$$
1+m_{1} m_{2} \cdots m_{k}\left\{-1+\sum_{i=1}^{k}\left(1-\frac{1}{m_{i}}\right)\right\} \text {. }
$$

This implies that $G$ is a free group of rank 4 and $H$ a free group of rank 5. Since $\Gamma^{\prime}$ is a free group of rank 2, Schreier's formula [3]

$$
R=1+\mu(r-1)
$$

for the rank $R$ of a subgroup of index $\mu$ in a free group of rank $r$ shows that $G$ is of index 3 in $\Gamma^{\prime}$ and $H$ of index 4 in $\Gamma^{\prime}$, completing the proof of the lemma.

LEMMA 2. We have

$$
\Gamma^{\prime}=G H .
$$

Proof. We note that the product is well-defined, since $G$ is a normal subgroup of $\Gamma^{2}, H$ a normal subgroup of $\Gamma^{3}$, and $\Gamma^{2} \supset \Gamma^{\prime}, \Gamma^{3} \supset \Gamma^{\prime}$ (see [1]). Hence $G$ and $H$ are normal subgroups of $\Gamma^{\prime}$.

Consider the chains

$$
\Gamma^{\prime} \supset G H \supset G, \quad \Gamma^{\prime} \supset G H \supset H .
$$

The first implies that $\left(\Gamma^{\prime}: G H\right) \mid 3$, and the second that $\left(\Gamma^{\prime}: G H\right) \mid 4$. Hence $\left(\Gamma^{\prime}: G H\right)=1$ and so $\Gamma^{\prime}=G H$, completing the proof of the lemma. (This version of the proof was suggested by the referee and editor.)

LEMMA 3. We have

$$
\left(\Gamma^{\prime}: \Gamma(6)\right)=12 .
$$

Proof. It is well-known that $\left(\Gamma: \Gamma^{\prime}\right)=6$ (see [1] for example) and that $(\Gamma: \Gamma(6))=72$. Since $\Gamma \supset \Gamma^{\prime} \supset \Gamma(6)$ the result follows.

We are now in a position to prove our result.

Theorem. We have

$$
\Gamma(6)=G \cap H \text {. }
$$

Proof. By one of the isomorphism theorems (since $G, H$ are normal subgroups of $\Gamma$ )

$$
G H / G \cong H / G \cap H .
$$

By Lemma 2 this reduces to

$$
\Gamma^{\prime} / G \cong H / G \cap H .
$$

Hence

$$
\left(\Gamma^{\prime}: G\right)=(H: G \cap H)
$$


and by Lemma 1 it follows that

$$
(H: G \cap H)=3 \text {. }
$$

Further, we have

$$
\left(\Gamma^{\prime}: G \cap H\right)=\left(\Gamma^{\prime}: H\right)(H: G \cap H)
$$

and again by Lemma 1 it follows that

$$
\left(\Gamma^{\prime}: G \cap H\right)=4(H: G \cap H) .
$$

Together with (2), (3) implies that

$$
\left(\Gamma^{\prime}: G \cap B\right)=12 .
$$

Since $G \cap H \supset \Gamma(6)$ (formula (1)) and $\left(\Gamma^{\prime}: \Gamma(6)\right)=12$ (Lemma 3) it follows that $\Gamma(6)=G \cap H$, completing the proof of the theorem.

\section{REFERENCES}

1. M. Newman, The structure of some subgroups of the modular group, Illinois J. Math. 6 (1962), 480-487.

2. J. Nielsen, The commutator subgroup of the free product of cyclic groups, Mat. Tidsskr. B. (1948), 49-56. (Danish)

3. O. Schreier, Die Untergruppen der freien Gruppen, Hamburger Abh. 5 (1927), 161-183.

4. J. R. Smart, Modular forms of dimension -2 for subgroups of the modular group, Thesis, Michigan State Univ., East Lansing, Mich., 1961.

National Bureau of Standards and NEW YORK UNIVERSITY 